\title{
Primera consulta sobre la industria de los materiales de la construcción organizada por ONUDI y UNCHS (HABITAT)
}

\author{
JULIAN SALAS, Dr. Ingeniero Industrial
}

IETCC/CSIC

\begin{abstract}
$R E S U M E N$
Se recogen en este trabajo, sobre la Primera Consulta patrocinada por ONUDI-UNCHS (HABITAT) relativa a la "Industria de los materiales de construcción", tres aspectos bien distintos sobre la misma: la versión definitiva de las conclusiones y recomendaciones elaboradas por los participantes en Atenas; las respuestas personales del autor, como participante, a algunas de las preguntas preparadas por los organizadores de la Consulta, asi como una breve reseña de las mesas redondas celebradas en el IETcc con algunos de los profesionales iberoamericanos participantes en el X Curso de Estudios Mayores de la Construcción centradas en este importante evento.

El objetivo, por tanto, es el de tratar de divulgar entre los lectores de habla hispana las recomendaciones de Atenas sobre el desarrollo de la industria de los materiales de construcción en los paises en vias de desarrollo.
\end{abstract}

\begin{abstract}
$S U M M A R Y$
This work, devoted to the First Consultation sponsored by ONUDI-UNCHS (HABITAT) relative to

"Building Materials Industry", selects three different sides of it: the final version of the conclusions and recommendations prepared in Athens by the participants, the author personal answers, as a participant himself, to some questions elabored by the Consultation organizers as well as a brief account of the round tables held in the IETcc with some of the Latin American professionals who took part in the X Course of High Studies on Construction focused on this important event.

Thus the aim is to try to spread among the readers of spanish language the Athens recommendations on the industry development of building materials in developing countries.
\end{abstract}

\section{INTRODUCCION}

La Primera Consulta sobre la Industria de los Materiales de Construcción se celebró en Atenas (Grecia), del 26 al 30 de marzo de 1985. Asistieron a la Consulta 160 participantes de 73 paises y 21 observadores de 16 organizaciones internacionales.

Del 15 al 17 de diciembre de 1982 se celebró en Viena (Austria) una reunión de expertos ad hoc sobre la industria de la construcción y de los materiales de construcción, a fin de examinar las conclusiones de los estudios preparados por la secretaria de la ONUDI sobre dicho sector e identificar los puntos y posibles temas de debate para la Primera Consulta.

Con objeto de asesorar a la ONUDI y al Centro de las Naciones Unidas para los Asentamientos Humanos (Hábitat) sobre la selección de los puntos prioritarios a considerar en la Primera Consulta, se celebró en Viena, del 24 al 28 de septiembre de 1984, una reunión global preparatoria de la Primera Consulta. La ONUDI y el citado Centro prepararon y presentaron a la reunión documentos de debate sobre posibles puntos a tratar. 
La reunión Global Preparatoria recomendó que en la Primera Consulta se considerasen los puntos siguientes:

\section{Punto 1}

Desarrollo de la industria de los materiales de construcción, incluidos los métodos de financiación, planificación y programación, con especial hincapié en los diferentes tamaños posibles de las plantas, particularmente en la industria del cemento.

\section{Punto 2}

Medidas encaminadas a fortalecer las capacidades tecnológicas nacionales para la producción de materiales de construcción, relacionadas, entre otras cosas, con la elección apropiada de productos, la selección, adaptación y transferencia de tecnología, la capacitación, la organización de la producción, el diseño y la producción de bienes de capital, y la investigación y el desarrollo.

\section{Punto 3}

Medidas necesarias para desarrollar la producción de materiales de construcción en el sector no estructurado de la economía, incluidos investigación y desarrollo, información, capacitación, y mejora de la calidad.

\section{PARTICIPACION EN LA CONSULTA}

Nuestras intervenciones personales, durante la Consulta, se centraron básicamente en los aspectos siguientes:

Respecto a ¿qué necesidades habrá de satisfacer la industria de los materiales de la construcción, en los países en desarrollo, dentro de los próximos años? Pensamos que en lo que resta de siglo, será sin duda la de su crecimiento cuantitativo. La miseria en el tema de vivienda a escala mundial hace esperar que esta reciba una atención superior a la del presente. La producción de materiales, productos, elementos y componentes constructivos deberá incrementarse de forma espectacular.

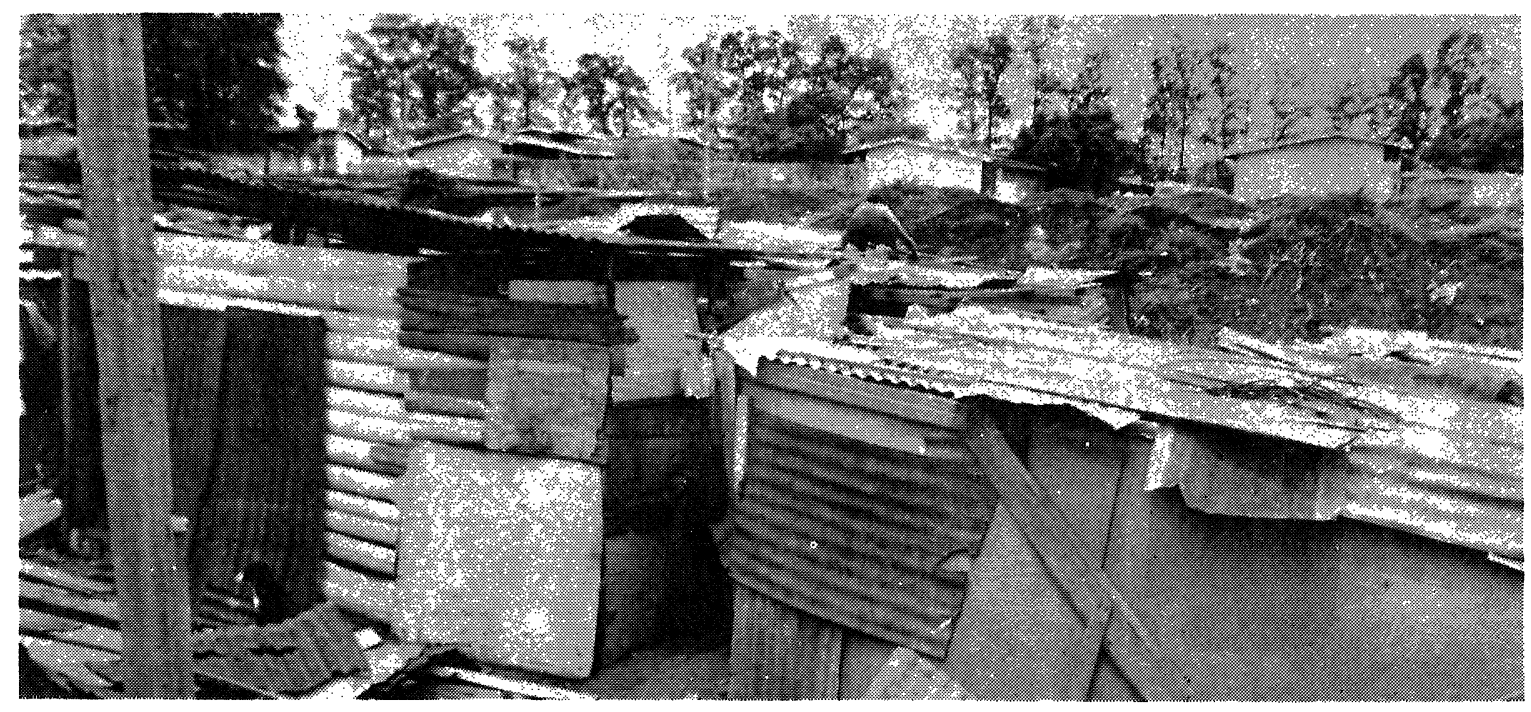


Otra característica de la evolución previsible de la industria de los materiales de construcción será la del empleo selectivo de los materiales que requieran un alto consumo energético o un proceso productivo intensivo en capital.

Resulta previsible una aceleración en el proceso ya en marcha por el que ciertos desechos se tranformaron en residuos, recientemente han pasado a ser subproductos y posiblemente antes de que finalice el siglo serán conocidos como materias primas. Nos estamos refiriendo a: los $400 \cdot 10^{6}$ de $\mathrm{Tm}$ anuales de cenizas volantes; $500 \cdot 10^{6} \mathrm{de}^{3}$ de cáscara de arroz; a las ingentes cantidades de relaves del cobre, residuos de bauxita, sedimentos y depósitos pantanosos, bagazo de caña de azucar, etc.

Ante el debate del si o no a las "listas básicas de materiales", defendimos que la confección de una lista básica de materiales de construcción necesarios en los paises en desarrollo, por completa y técnicamente bien planteada que fuese, no nos parece ni oportuna ni adecuada. Sería, cuando menos, resultado de una visión desde el desarrollo de los problemas de Tercer Mundo.

Más valida nos parece la elaboración de una lista de "performances" funcionales, valores minimizados en extremo, capaces de reflejar distintos escenarios muy concretos: viviendas permanentes de una planta en zona húmeda-sísmica; viviendas crecederas en zona tropicalciclónica; etc.

Otro tema abordado fue el de la importancia de la escala en el sector, así como posibilidades y limitaciones de la fabricación en pequeña escala. Hemos mantenido al respecto que estamos ante un sector típico de demanda insatisfecha. En los paises en desarrollo se trata de una demanda básicamente cuantitativa, que deviene cualitativa según aumenta el nivel de ingresos. Si se tiene presente únicamente la demanda solvente, la escala puede ser importante en el desarrollo del sector. Si lo que se tiene en cuenta es la necesidad objetiva, la escala presenta un papel insignificante.

Pese a que el sector construcción es un típico ejemplo de mezcla tecnologica, -incluso en paises muy desarrollados de mercado libre se dá una convivencia competitiva de tecnologías de índice de industrialización muy dispar-, es nuestra opinión, que en los países en desarrollo: por la baja incidencia de la mano de obra; el reducido radio de acción de las plantas por la escasez de vías de comunicación y medios de transporte adecuados; la existencia de una demanda intermitente, estacionaria, cíclica - capacidad de adecuación-; la consecución de calidad suficiente con procesos de escasa inversión; etc. hacen que la pequeña escala (en los rubros productivos en que

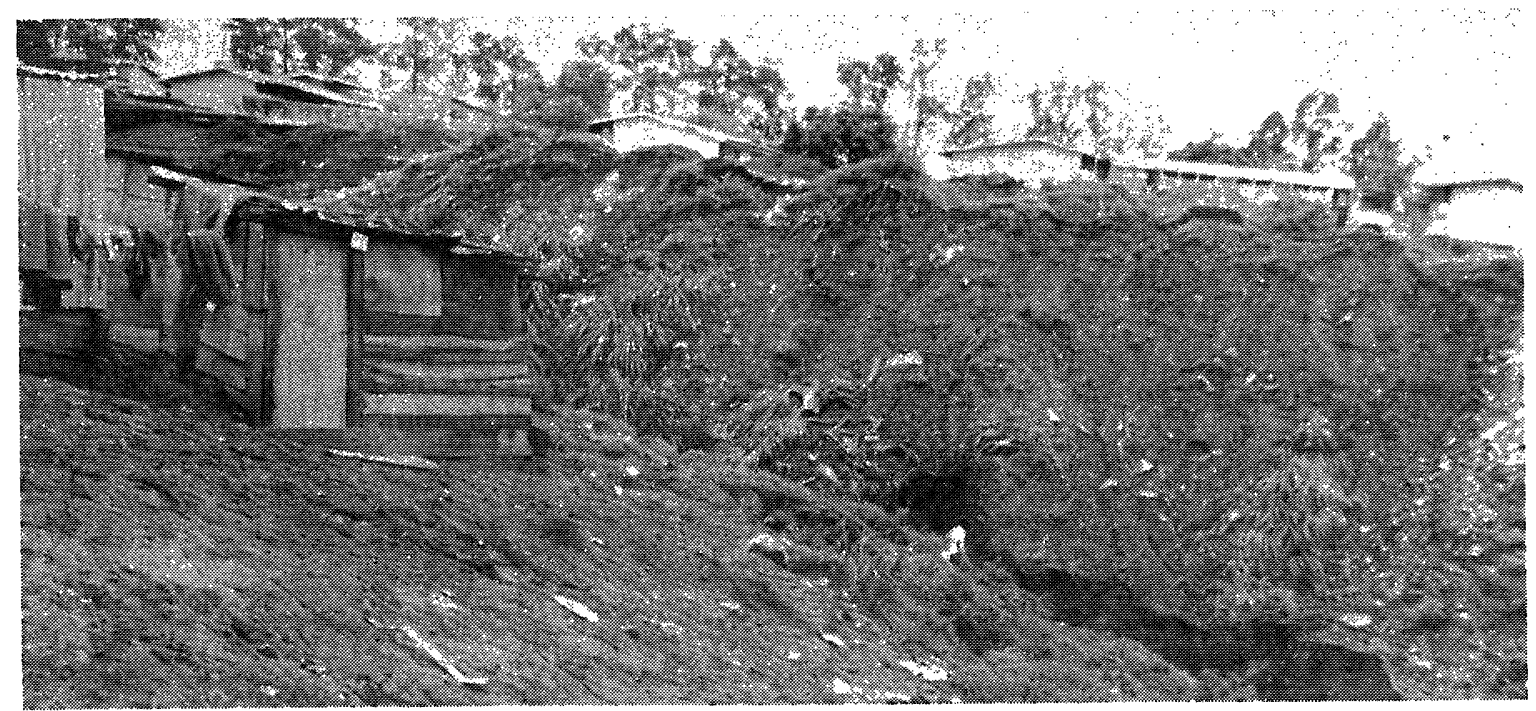


ello es factible) se acomode mejor que la gran escala a las condiciones tipicas de los paises en dearrollo.

Mediante la Consulta se intentó conocer las posibilidades de cooperación internacional para mejorar las Normas y el Control de Calidad de los países en desarrollo.

Nuestra intervención trató de exponer que se detecta una clara tendencia de evolución de las antiguas pretensiones normativas con vocación: universal, exigencial, impositiva y polivalente (carácter maximalista), hacia otras, de intención: nacional, funcional, voluntaria y especifica sectorial (vivienda rural, bloques, ... estructuras de hormigón, metálicas, de madera...) con un sentido marcadamente posibilista.

La labor normativa, en todo caso, pero más aún en los paises en desarrollo, debe ser una labor realizada "in situ". Malas consecuencias se han derivado en no pocos casos de la simple adopción o traducción de normas foráneas. La cooperación internacional puede en este campo prestar un excelente servicio a los paises en desarrollo, siempre que se cumplan las siguientes condiciones:

a) Clara independencia de los cooperantes ante posibles opciones con implicaciones económicas.

b) Elaboración sobre el terreno, en contacto y propiciando la participación de instituciones, profesionales, y empresarios del país.

c) Suficiente divulgación del texto normativo que en no pocos aspectos debe tener un carácter informativo-pedagógico.

El Instituto Eduardo Torroja, puede aportar una reciente (1982-83) cooperación con Bolivia, de las caracteristicas enunciadas que dio como resultado la redacción del "Código Boliviano del Hormigón Armado C.B.H.-84".

Muy esquemáticamente, y por tanto de forma muy parcial, diriamos que es preferible una "mala" normá asumida y acorde con la realidad del país, que contar con un "excelente" corpus normativo ignorado por la realidad del sector construcción.

Se interrogaba, a los participantes en la Consulta de Atenas, sobre ¿Cómo pueden los gobiernos nacionales proporcionar un marco para elevar al máximo las contribuciones de la producción de

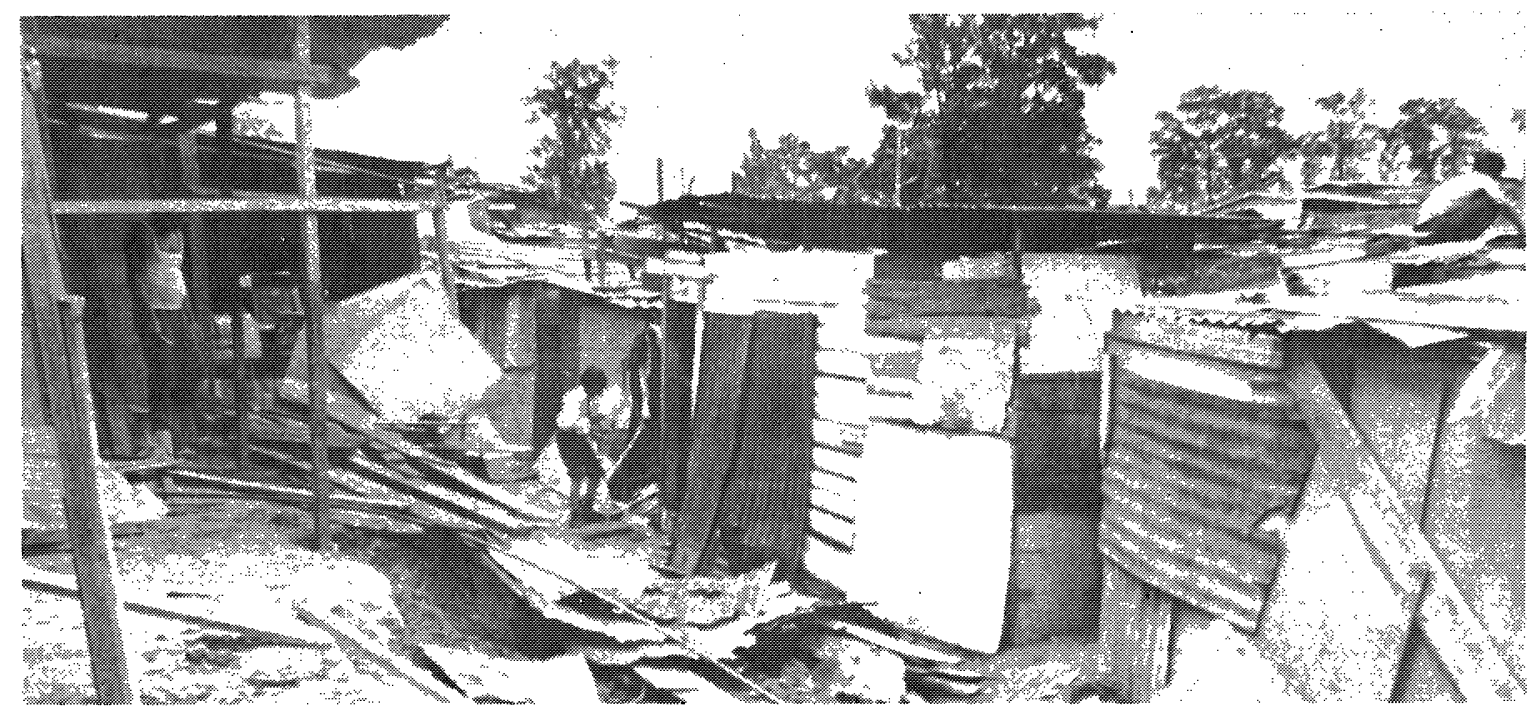


materiales de construcción en pequeña escala en la economía no estructurada, incorporando asistencia técnica o apoyo externos de la manera más eficiente?

La respuesta no puede ser única. Son muchas las variables que influyen en el sector de la vivienda y sus materiales como hemos podido constatar, una vez más, en la amplia y dispar respuesta obtenida al Concurso de Trabajos sobre el tema "Soluciones actuales al problema de la Vivienda en Iberoamérica" patrocinado por nuestro Instituto.

Nos centraremos, por su implantación y excelentes resultados en contextos tan diversos como Perú, Cuba y Argențina, a la práctica conocida como "banco de materiales" (Perú), "venta modulada de materiales a la población" (Cuba), "crédito para materiales" (Argentina). Consiste básicamente en un sistema de crédito por el cual se otorgan préstamos a medio-largo plazo destinados exclusivamente a la adquisición de una lista predeterminada de materiales suficientes para construir una sencilla vivienda de unos $30 \mathrm{~m}^{2}$. La Institución entrega directamente los materiales que el solicitante ha escogido de una lista básica, otorgando préstamos que no cedan de una cierta cantidad $(\simeq 1.000 \$)$ acompañada en algunos casos de asistencia técnica.

El procedimiento responde en un doble aspecto a la cuestión formulada. De una parte, contribuye a la producción de viviendas en el sector no estructurado, por otra, la proliferación de "una lista básica de materiales de construcción" supone la aparición de una próspera red descentralizada de pequeños productores de elementos, capaces de atender unos niveles cualitativos adecuados establecidos por la entidad financiadora.

Como respuesta parcial a una pregunta, sobre el apoyo que la comunidad internacional puede prestar a las cualificaciones profesionales, dejamos constancia de la importancia que para los países en desarrollo tiene el que las organizaciones internacionales ayuden a que los profesionales y las instituciones donde se forman, no den la espalda al estudio y la práctica de los materiales y tecnologias autóctonas. Un equivoco concepto de "modernidad" en los estudios universitarios de muchos paises en vias de desarrollo puede llevarlos a situaciones sin salida.

Acciones concretas, como la excelente exposición "Arquitecturas de Tierra o del Porvenir de una Tradicción Milenaria", preparada por el centro "Georges Pompidou" (París 1981-82), pueden ser valiosas aportaciones en el sentido antes apuntado. Una llamada a las coincidencias de los profesionales de los paises donde el adobe y el tapial fue una herramienta, hoy semiabandonada, y que puede prestar aún excelentes servicios.

De la Consulta de Atenas también sacamos como conclusión personal - tangencial con el tema

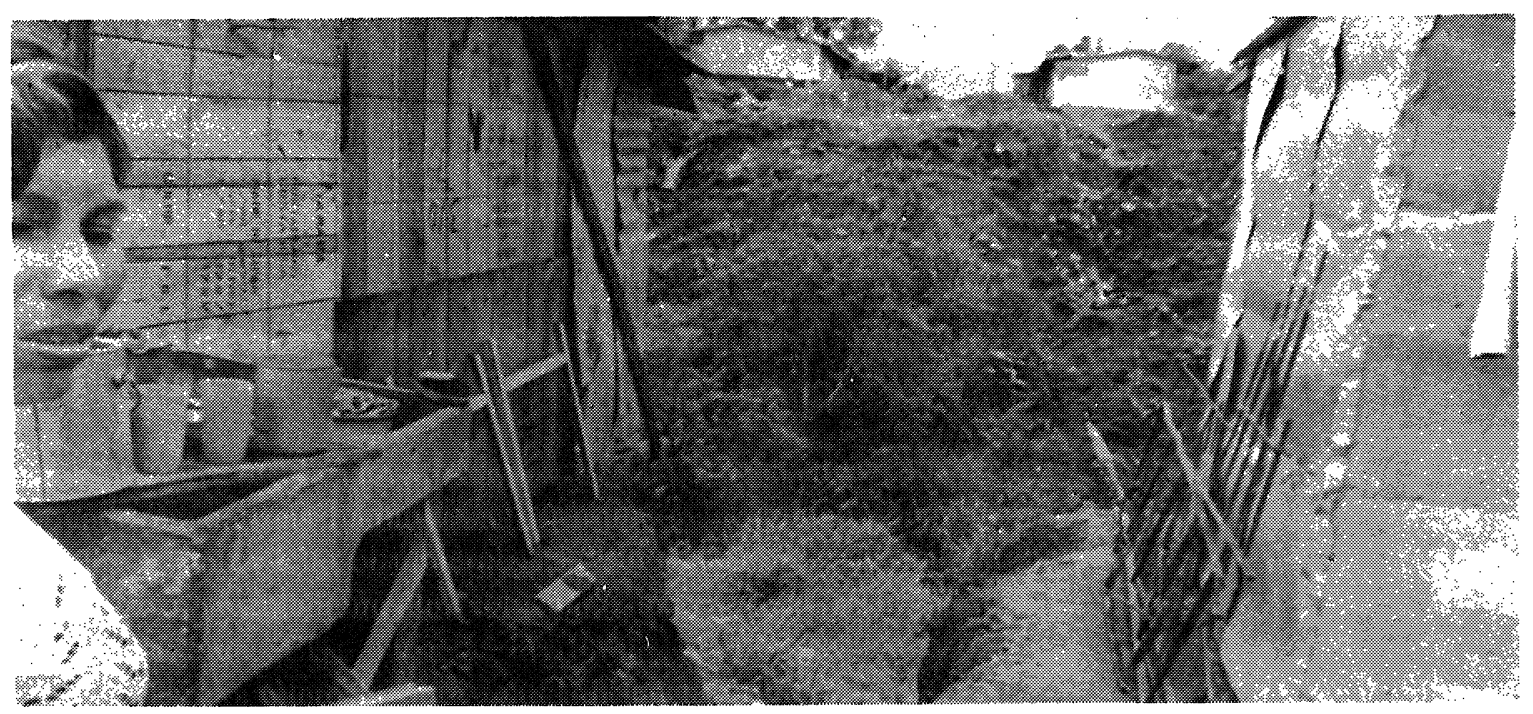


de la misma - el constatar, una vez más, la necesidad de que una institución nacional se ocupe de la coordinación e intensificación de nuestra Cooperación Internacional a todos los niveles y, muy particularmente, con Iberoamérica.

\section{DEBATE SOBRE LOS TEMAS DE LA "CONSULTA" CON PARTICIPANTES EN EL X CEMCO}

\author{
Lista de participantes \\ EMHA MATILDE AMADO (Arquitecta. Argentina). \\ MARINA ALVARFZ (Arquitecta. IETcc). \\ LIANA ARRIETA BUSTILLOS (Ingeniera Civil. Venezuela) \\ JOSE MIGUEL BARBERO (Arquitecto. IETcc). \\ RUBEN BURBANO (Ingeniero Civil. Argentina). \\ MARIA ANTONIETA CARBONI (Arquitecta. Costa Rica). \\ CARLOS DE LA CUESTA (Ingeniero Civil. Colombia). \\ PABLO DIAZ-ROMERAL (Arquitecto. IETcc). \\ JUAN DIAZ-ROMERAL (Aparejador. IETcc). \\ DANTE DOMENE. Redactor (Arquitecto. Argentina). \\ PABLO GONZALEZ ANTEZANA (Arquitecto. Chile).

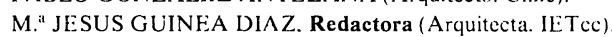

LUIS FERNANDO LIAN (Ingeniero Civil. Colombia) MIRIAN LOPEZ (Ingeniera en Construcciones. Argentina). PILAR LOZANO RICO (Arquitecta. Colombia).

NOEMI GRACIELA MALDONADO (Ingeniera en Construcciones. Argentina). GIOCONDA POVEDA ARAGUNDI (Ingeniera Civil. Ecuador).

JULIAN SALAS SERRANO. Redactor (Dr. Ingeniero Industrial. IETcc).

ALEJANDRO SALAZAR (Ingeniero Quimico. Colombia).

M. ${ }^{a}$ ISABEL SANCHEZ DE ROJAS, Redactora (Lcda. en C. Quimicas. IETcc).

ENRIQUE SERVIDE (Arquitecto. Argentina).

FERNANDO TRIVIÑO (Dr. en Ciencias Quimicas. IETcc).

TOMAS VAZQUEZ MORENO (Dr. en Ciencias Quimicas. IETcc).

JANER VERAS CASTRO (Ingeniero Civil. República Dominicana).

Dado el interés que suscitó la primera Consulta sobre el tema de la industria de los materiales de construcción, alumnos del Curso CEMCO (Curso de Estudios Mayores de la Construcción) y miembros del Equipo de V.M.B.C. (Viviendas de muy bajo coste), organizaron una mesa redonda de tres sesiones con los participantes que se relacionan en el cuadro a pie de página, con objeto de comentar y tratar de dar una respuesta a la Consulta de la ONUDI, con algunas aportaciones propias y nuevas ideas que añadir a las conclusiones a que se llegaron en Atenas, asi como propiciar la divulgación de las mismas a través de los distintos Ministerios de los paises iberoamericanos, como paises en vías de desarrollo.

El principal problema en Iberoamérica, para la industria de los materiales de construcción, es la falta de medios económicos, la financiación y el endeudamiento a que se ha llegado con el Fondo Monetario Internacional. Se origina, también, un problema de planificación y politico hasta conseguir el asentamiento de las democracias; dados los cambios politicos, se paralizan todas las decisiones y no duran más de dos años los ciclos de planificación. Sólo subsisten, en estos periodos, las grandes empresas y los pequeños artesanos. Quien decide en algunos de estos paises no es quien conoce el problema, y esto es grave, para lo cual se debe hacer una llamada de atención a los planificadores con objeto de que planifiquen con sentido práctico.

Las dos grandes potencias mundiales han tomado conciencia de que el problema de la vivienda ha llegado a una situación explosiva, y no se puede resolver éste sin antes no haber solucionado el de la industria de los materialcs. La ONUDI va directamente, con esta Consulta, a las raices; no es un problema de arquitectura ni de sociologia.

La industria de los materiales deberia tener un gran crecimiento cuantitativo para paliar el problema de la escasez de viviendas a escala mundial, siempre con el apoyo del poder politico, sin paternalismos y con ayudas financieras y burocráticas.

Gran parte de los paises en vias de desarrollo no tienen un conocimiento claro de sus propias materias primas y de sus necesidades, por lo que seria conveniente un estudio exhaustivo de las mismas, asi como una divulgación del conocimiento y uso de estos materiales autóctonos, que serán abundantes en cada pais y casi gratuitos, lo que proporcionaria poder sustituir parte de los materiales tradicionales por otros de recursos locales para conseguir una menor dependencia económica, así como el empleo selectivo de los materiales que no requieran un alto consumo cnergético y un proceso productivo muy tecnológico. Se buscarán aquellos productos o materias 
primas que antes eran desechos, después residuos y que, posteriormente, pasaron a ser subproductos, tales como han sido las cenizas volantes, la cáscara de arroz, el bagazo de caña de azúcar, residuos de la bauxita, etc.

Se deben buscar soluciones mediante un tipo de industria básica, lo más autóctona posible, y pequeñas factorías de poco rendimiento económico, pero de gran rendimiento social, que promueven gran número de mano de obra y ahorran transporte.

Es importante la cuestión de la escala para el desarrollo de este sector, sin ser una competencia entre grandes y pequeñas fábricas. El tamaño estaría de acuerdo con la estructura de cada pais, como puede ser la red de carreteras o la ubicación de las ciudades y pueblos, llegándose a una combinación para lo cual las dos soluciones no son excluyentes.

Hoy día, las pequeñas industrias tienen un gran campo de aplicación debido a la crisis económica, el aumento del paro y la mano de obra. Es positivo el desarrollo de estas pequeñas industrias y de las pequeñas factorías artesanales - abundantes en Iberoamérica-, pero bien planificadas y con ayudas financieras. Esto se puede conseguir con una cooperación in situ con técnicos formados y con plena conciencia del problema, consiguiendo una continuidad de los programas y no tener que llegar a la improvisación. Siendo, más tarde, las experiencias adquiridas transmitidas a usuarios con problemas similares del mismo pais o de otros.

Se debe crear en los paises una conciencia de la importancia de la investigación con buenos programas a desarrollar, sin duplicarse ni repetirse. Se podria conseguir con:

1) Existencia de un Banco de Datos en español con terminales, por lo menos, en todas las capitales de los paises iberoamericanos.

2) Organización de ayuda mutua iberoamericana (al igual que el Mercado Europeo).

3) Creación de una revista internacional de habla hispana, con un comité formado por integrantes de diferentes países, consiguiendo refundir el gran número de revistas dedicadas a este tema en una sola y buena.

Dentro de la investigación de los materiales es necesario llegar a un control de la calidad de los mismos con la redacción de una normativa clara, real y no hipotética; es decir, de acuerdo con los materiales del pais.

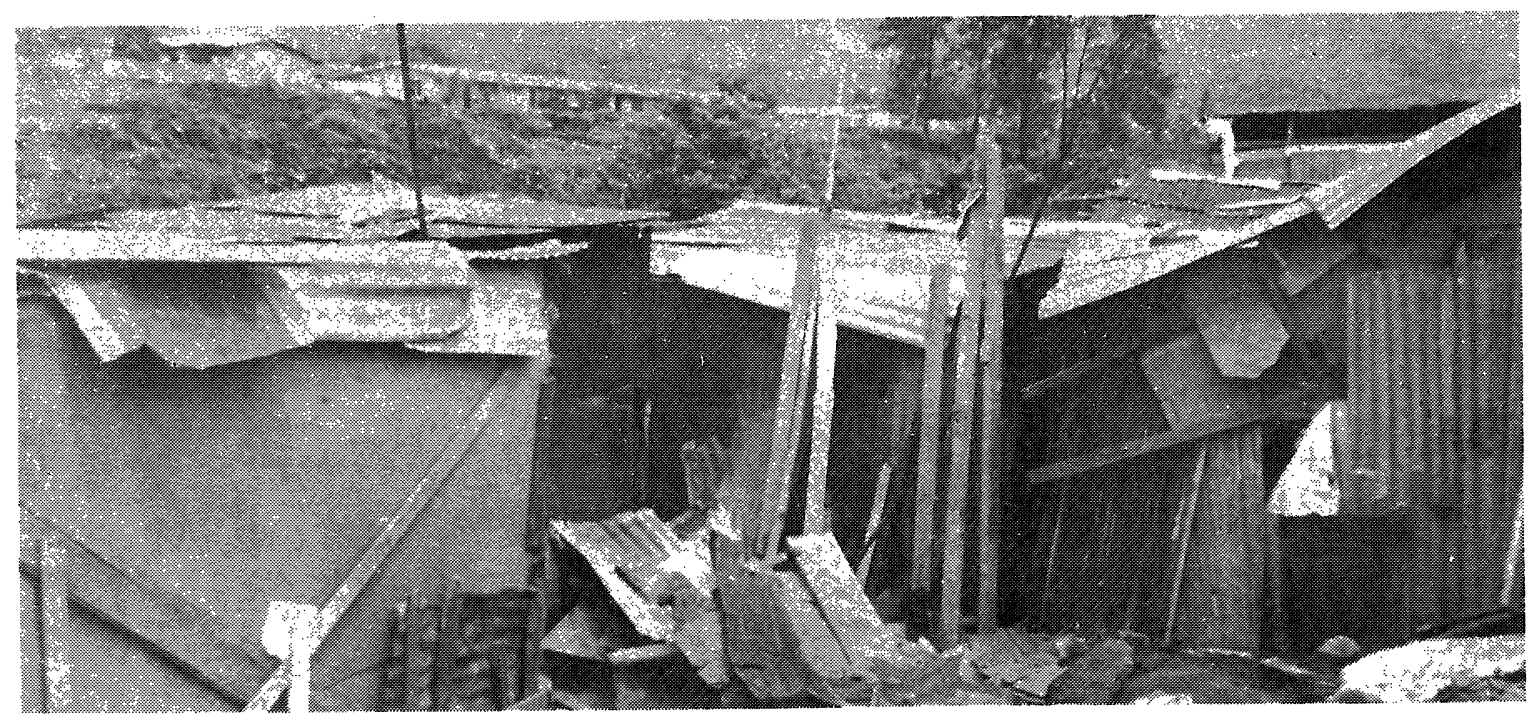

Fotos Arqta. M. GARBONI. 
Sin la ayuda del sector no estructurado, de aquel que por sus propios medios llega a la creación de su vivienda, es decir, por autoconstrucción, no hubiera sido posible paliar el "déficit de la vivienda", ya que, por lo menos, la cuarta parte de este problema la está resolviendo este sector $y$, por tanto, deberia tener un apoyo estatal. Algunos Gobiernos han tomado conciencia de ello creando:

1. Centros de asesoramiento de tecnologias y materiales apropiados.

2. ${ }^{\circ} \quad$ Créditos programados para la adquisición de materiales.

3. ${ }^{\circ}$ Bancos de Materiales.

4. ${ }^{\circ}$ Cooperativas.

5. ${ }^{\circ}$ Mayor protagonismo de los sindicatos que conocen el problema.

El coloquio de Atenas ha podido ser un intento de concienciación al más alto nivel con objeto de que, de aquí a un año, volver a incidir en el tema.

\section{Conclusiones y recomendaciones acordadas}
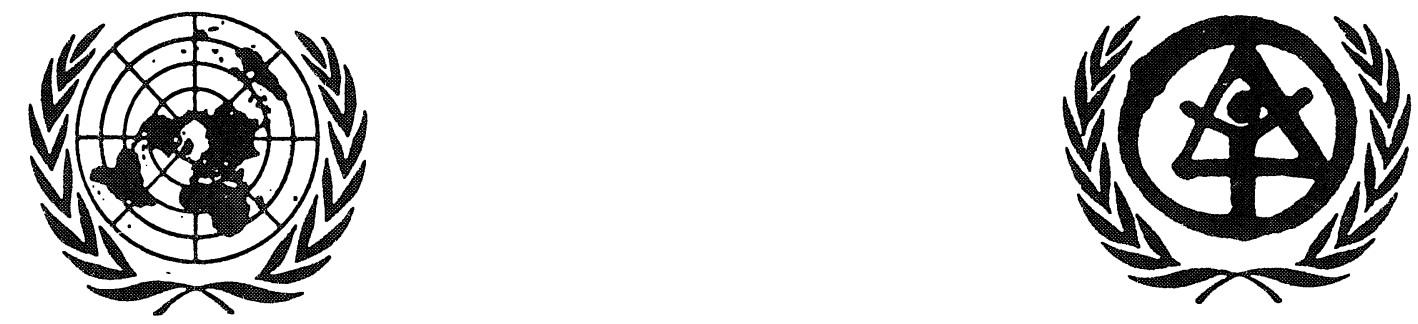

Organización de las naciones unidas

para el desarrollo industrial

Centro de las Naciones Unidas para los asentamientos humanos (HABITAT)

\section{PUNTO 1: Desarrollo de la industria de los materiales de construcción, y}

\section{PUNTO 2: Medidas encaminadas a fortalecer las capacidades tecnológicas nacionales para la producción de materiales de construcción}




\section{PLANIFICACION}

\section{Conclusión}

La Primera Consulta llegó a la conclusión de que era necesario estimar las necesidades de materiales de construcción y efectuar previsiones a largo y mediano plazo de su demanda, así como que los países en desarrollo hicieran inventarios de los recursos existentes y potenciales en cuanto a materias primas, fuentes energéticas, infraestructura y personal calificado, técnicas y capacidades locales disponibles, lo cual permitiria la transformación de esas materias primas en materiales de construcción. Con tal fin debian formularse politicas adecuadas y planes a largo plazo que tuvieran la flexibilidad necesaria para poder introducir ajustes a corto plazo.

\section{Recomendaciones}

La ONUDI debería ayudar a los países en desarrollo a mejorar sus sistemas de planificación con respecto a la industria de los materiales de construcción, particularmente en esferas como las siguientes:

a) Fortalecimiento de los sistemas de información para el establecimiento de inventarios de recursos, la estimación de las necesidades y la previsión de la demanda. Tales sistemas deberían ser accesibles a todos los usuarios, especialmente a las empresas productoras;

b) Elaboración de estrategias a largo plazo encaminadas a aumentar la oferta de materiales de construcción y a ampliar su variedad a base de los recursos locales;

c) Mejoramiento de los métodos de planificación y de la organización con miras a identificar los obstáculos y coordinar esfuerzos, en particular los relativos a la coordinación con otros sectores conexos;

d) Intensificación de la cooperación regional en coordinación con las comisiones y organismos regionales.

\section{ESCALA DE PRODUCCION Y TECNOLOGIA}

\section{Conclusión}

La Primera Consulta llegó a la conclusión de que los paises en desarrollo debian tener mayor acceso a la tecnología más apropiada a sus condiciones socioeconómicas. Convino en que no era posible demostrar la superioridad de una determinada tecnología o escala de planta sobre otra. Por consiguiente, los países en desarrollo debían tener acceso a la información disponible sobre las condiciones técnicas y económicas de diversas tecnologías, a fin de poder efectuar la mejor elección al adquirir equipo para plantas. 


\section{Recomendaciones}

La Primera Consulta recomendó que:

a) La ONUDI proporcionara a los países en desarrollo que lo soliciten información actualizada, a través del Banco de Información Industrial y Tecnológica (INTIB), sobre las principales opciones tecnológicas para la producción -en diferentes escalasde materiales de construcción, así como sobre las fuentes de información más detallada;

b) Se invitase a los gobiernos y a las organizaciones no gubernamentales a que informaran a la ONUDI sobre las tecnologias de los materiales de construcción que estuvieran dispuestos a compartir con los países en desarrollo.

\section{FINANCIACION}

\section{Conclusiones}

La Primera Consulta llegó a la conclusión de que la isuficiencia de la financiación externa y local constituía un obstáculo importante para el desarrollo del sector. Estuvo de acuerdo en que algunas instituciones financieras no solían tomar en cuenta la financiación de la adquisición de repuestos, de los programas de capacitación ni del capital de explotación. Recalcó la necesidad de examinar los problemas financieros tanto de la industria de la construcción como de la producción de materiales de construcción, y de hallar los medios de movilizar y dirigir el ahorro interno. También convino en que, para lograr la industrialización de los paises en desarrollo en el sector de los materiales de construcción, una forma útil de cooperación que había que estimular más era la inversión directa, por la que los empresarios se corresponsabilizaban de la eficacia, la rentabilidad, el seguimiento y el éxito de las operaciones.

\section{Recomendaciones}

La Consulta recomendó que:

a) Se invitara a las instituciones bancarias internacionales y nacionales a que concediesen al sector una prioridad acorde a su importancia estratégica para la economía. Dichas instituciones debian también considerar la posibilidad de conceder la debida importancia a la financiación de plantas, a la adquisición de piezas de repuesto, a los programas de capacitación y al capital de explotación;

b) Se abrieran líneas de crédito especiales para la rehabilitación de plantas ya existentes y para la promoción de nuevos procesos y pequeñas unidades con destino a la producción de materiales de construcción.
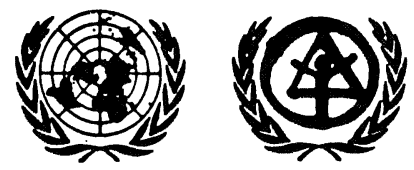


\section{INVESTIGACION Y DESARROLLO}

\section{Conclusiones}

La Primera Consulta llegó a la conclusión de que era necesario:

a) Incrementar las actividades de investigación tecnológica y la vinculación entre las instituciones de investigación y la industria de los materiales de construcción;

b) Seguir explorando los recursos minerales - y otros recursos- locales para la producción de materiales de construcción;

c) Realizar un inventario de otras materias primas y de otros tipos de energia que pudieran utilizarse en la producción de materiales de construcción;

d) Emprender la investigación y el desarrollo de esos recursos en cooperación con todas las partes interesadas;

e) Aumentar la investigación de los productos para mejorar su calidad, teniendo en cuenta las necesidades de utilización y aplicación finales;

f) Intensificar la participación de los centros de investigación y desarrollo existentes, en los planos nacional e internacional, en el desarrollo de procesos tecnológicos mejorados y del equipo correspondiente, deberia basarse en los recursos disponibles en los paises en desarrollo teniendo en cuenta su potencial financiero y humano;

g) Aumentar la corriente de información sobre actividades de investigación entre todas las partes interesadas.

\section{Recomendaciones}

La Primera Consulta recomendó que la ONUDI, en cooperación con las organizaciones internacionales y con otros organismos interesados, promoviera las actividades de investigación concernientes a la industria de los materiales de construcción en las siguientes esferas prioritarias:

a) Recursos nacionales de materias primas y su idoneidad para la producción de materiales de construcción;

b) Investigación y desarrollo de productos, teniendo en cuenta el empleo final de los materiales;

c) Ensayo y evaluación de tecnología y de materiales de construcción adecuados. También debía estimularse la cooperación en el fortalecimiento de instalaciones de ensayo ya existentes o en el establecimiento de otras nuevas.

La Primera Consulta recomendó una mayor participación, a nivel gubernamental y no 
gubernamental, de los centros de investigación y desarrollo nacionales e internacionales, a fin de promover una mejor utilización de los recursos locales disponibles, el mejoramiento de las capacidades de producción existentes, el ahorro de energía y la sustitución de combustibles.

La Primera Consulta recomendó que los gobiernos estableciean o fortaleciesen servicios e instalaciones institucionales para la investigación el desarrollo y la información sobre la construcción y los materiales de construcción. Los gobiernos y la ONUDI debian cooperar con las asociaciones internacionales científicas y técnicas relacionadas con las industrias de la construcción y de los materiales de construcción, a fin de facilitar la participación de los países en desarrollo en las actividades de esas asociaciones.

\section{NORMAS, CODIGOS Y REGLAMENTACIONES}

\section{Conclusión}

La Primera Consulta llegó a la conclusión de que era necesario que los países en desarrollo adaptasen normas, códigos y reglamentaciones a sus circunstancias socioeconómicas, a sus niveles técnicos y a su medio ambiente.

\section{Recomendaciones}

Se recomendó que los países en desarrollo establecieran un marco de reglamentaciones basadas en las de las organizaciones nacionales, regionales o internacionales, y que las adaptasen a sus necesidades.

También se recomendó que la ONUDI y el Centro de las Naciones Unidas para los Asentamientos Humanos (Hábitat), en cooperación con organizaciones competentes, y teniendo en cuenta la labor que se realizaba en esta esfera, promovieran el desarrollo de normas, códigos y reglamentaciones - orientados a los niveles de exigencias- que se ajustasen a las necesidades concretas de los países en desarrollo en la esfera de la construcción y el empleo de materiales locales de construcción, procurando al mismo tiempo conseguir un grado esencial de normalización a los niveles regional e internacional.

\section{CONSULTORIA, SELECCION DE TECNOLOGIAS}

\section{Conclusiones}

La Primera Consulta llegó a la conclusión de que se debía recurrir en mayor medida a las capacidades de consultoría existentes en los paises en desarrollo.

También se llegó a la conclusión de que los paises en desarrollo necesitaban asistencia en la evaluación de ofertas y en la selección de tecnologías. Se reconoció que la falta de capacidades en materia de servicios consultivos podía ocasionar graves errores a la hora de elegir tecnología, con el resultado de un mal funcionamiento de las plantas, un costo excesivo y retrasos en la puesta en marcha.

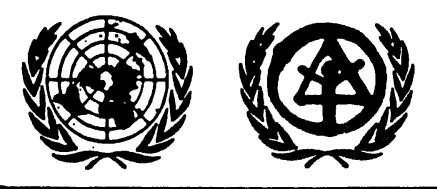




\section{Recomendacion}

La Primera Consulta recomendó que la ONUDI mantuviera, y a ser posible fortaleciese, sus actividades de capacitación en cuanto a la evaluación de ofertas, la selección de tecnologias y las técnicas de negociación.

\section{COOPERACION, CAPACITACION}

\section{Conclusiones}

La Primera Consulta llegó a la conclusión de que era necesario:

a) Aumentar los esfuerzos de capacitación, tanto individual como colectiva, a todos los niveles técnicos, con especial hincapié en la capacitación en el trabajo;

b) Aprovechar las fuentes de capacitación que ofrecía la industria privada y que hasta entonces no se habian utilizado;

c) Capacitar a instructores.

\section{Recomendaciones}

La Primera Consulta recomendó que los gobiernos de los paises en desarrollo elaboraran políticas para estimular y mejorar la capacitación, a todos los niveles técnicos, en la producción de materiales de construcción.

También se recomendó que la ONUDI prestara asistencia a los gobiernos de los países en desarrollo para que aprovecharan las posibilidades de capacitación que ofrecian los sectores público y privado de los paises desarrollados y de los paises en desarrollo.

\section{PUNTO 3: Medidas necesarias para desarrollar la producción de materiales de construcción en el sector no estructurado de la economía, incluidos investigación y desarrollo, información, capacitación, y mejora de la calidad}

\section{Conclusiones}

La Primera Consulta llegó a la conclusión de que:

a) El sector no estructurado de los materiales de construcción contribuía de manera importante - sobre todo en los paises en desarrollo- al crecimiento de la industria y al desarrollo económico nacional, mediante vinculaciones con otros sectores de la economía, y los productores del sector no estructurado podian ampliar sus actividades mediante un proceso de crecimiento incremental; 
b) Debido principalmente a la falta de apoyo, la producción de materiales de construcción en el sector no estructurado tropezaba con serias limitaciones en cuanto a la escasa calidad de los productos, la limitada gama de éstos, la insuficiente oferta y, a veces, el elevado costo de la producción;

c) Los gobiernos de los países en desarrollo tenían una importante función que desempeñar en la constitución de un marco para promover y mejorar el sector no estructurado de materiales y elementos de construcción, incluidos todos los artículos necesarios para la industria de la construcción, mediante la integración de esos sectores en una política de construcción y viviendas. Esto podría realizarse especialmente por ser ellos los principales clientes de la industria de los materiales de construcción. En particular, los gobiernos podian proporcionar apoyo adquiriendo productos del sector no estructurado, movilizando el ahorro para financiar la provisión de bienes de capital, capital de explotación y créditos, y prestando apoyo a organizaciones locales para la difusión de información sobre tecnologias apropiadas a dicho sector. Las instituciones y organizaciones locales relacionadas con el sector, así como las personas directamente interesadas, deben participar plenamente en estas actividades gubernamentales $\mathbf{y}$ en otros tipos de actividades análogas;

d) Las reglamentaciones y códigos de construcción apropiados podrian ser instrumentos importantes, pero eran muy dificiles de aplicar en el sector no estructurado. A fin de superar este obstáculo, era necesario considerar enfoques pragmáticos, especialmente la producción de diseños tipo, la adopción de reglamentaciones deseadas en los proyectos de demostración y la promoción de normas y especificaciones para orientar a los productores del sector no estructurado;

e) Los gobiernos disponian de una amplia serie de instrumentos que podrían ser utilizados en la aplicación de una política para estimular al sector no estructurado mediante licitaciones, proyectos de construcciones públicas, y políticas de compras y de financiación. Los gobiernos también tenían un papel que desempeñar en el apoyo a la realización de estudios básicos del sector $\mathrm{y}$ en el establecimiento de proyectos de demostración y programas de investigación universitarios apropiados;

f) La comunidad internacional podría ayudar a los gobiernos a promover la producción de materiales y elementos de construcción del sector no estructurado, especialmente en materia de transferencia y desarrollo de tecnologías, promoción de métodos de mejoramiento de la calidad, programas para el perfeccionamiento profesional básico en los planos técnico y de gestión, y apoyo a estudios empíricos. Instrumentos adecuados serían, por ejemplo, la difusión de datos disponibles, la ayuda a los centros locales de adaptación de tecnología, y la promoción de la elaboración y de la difusión de normas y de reglas apropiadas, fundadas preferiblemente en el enfoque de los niveles de exigencia en lugar de en el enfoque de la especificación de materiales;

g) La comunidad internacional podria apoyar o emprender estudios sistemáticos del sector, empezando por un examen y un análisis de los estudios e informes existentes con miras a identificar las lagunas en los conocimientos, formular directrices para la recopilación y el análisis de datos y realizar, en su caso, estudios específicos, que deberían servir de base al establecimiento de estrategias de desarrollo realistas.

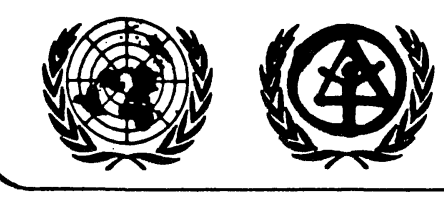




\section{Recomendaciones}

La Primera Consulta recomendó que los gobiernos de los paises en desarrollo elaboraran políticas para estimular y mejorar el desarrollo de un sector no estructurado de los materiales de construcción, con lo que contribuiría a aumentar la producción de este sector y el empleo de sus productos. A tal fin, se invitó a esos gobiernos a:

a) Reconocer la importancia de la producción de elementos y materiales de construcción por parte del sector no estructurado y prestar pleno apoyo a los proyectos encaminados al desarrollo de este sector;

b) Facilitar, en los proyectos de construcción estatales, el empleo de materiales de construcción producidos por el sector no estructurado; por ejemplo, para edificaciones de tipo sencillo en las que dichos materiales pudieran dar resultado satisfactorio. A fin de proporcionar al sector no estructurado un máximo de posibilidades de suministrar estos materiales y elementos, los documentos contractuales y de licitación deberian estar concebidos de manera que fuesen fáciles de entender y de formalizar;

c) Tomar las medidas necesarias para que las viviendas y otras instalaciones fueran concebidas de modo que favoreciesen la utilización de materiales y elementos del sector no estructurado;

d) Alentar la aceptación y, en su caso, la elaboración de pautas, o tal vez incluso de normas y especificaciones que permitiesen el empleo de materiales y elementos producidos por el sector no estructurado, y englobar sistemáticamente estos materiales y elementos en las reglamentaciones de construcción mediante el concepto de niveles de exigencia;

e) Fortalecer las actividades de las instituciones locales o, si fuera necesario, establecer instituciones locales que se centraran en la aplicación práctica de la investigación, como, por ejemplo, en el marco de proyectos de demostración y para el control de calidad, y reforzar los vínculos entre las actividades de investigación internacionales y nacionales y el sector no estructurado;

f) Ayudar a movilizar el ahorro mediante el reconocimiento de las sociedades de ahorro tradicionales o el estímulo al desarrollo de las asociaciones de crédito y ahorro oficialmente constituidas o no; prestar apoyo financiero, por ejemplo, a bancos de acopio y de materiales; proporcionar a estas sociedades facilidades para la importación del equipo indispensable y promover el establecimiento de organizaciones de arriendo de equipo; e invitar a los bancos a que también presten servicios al sector no estructurado;

g) Identificar y proporcionar apoyo a todas las organizaciones locales, incluso a las que no estuviesen especializadas en el sector de los materiales de construcción, que pudieran servir de vectores para difundir información entre los productores del sector no estructurado, por ejemplo, sobre tecnologías sencillas y métodos de control de calidad; 
h) Apoyar los esfuerzos tendientes a proporcionar en el plano municipal, en el marco de proyectos de vivienda y de construcción, el espacio y los medios necesarios para que el sector no estructurado pueda producir materiales y elementos de construcción.

La Primera Consulta recomendó que se invitara a la comunidad internacional —entendida como el conjunto de organizaciones internacionales gubernamentales y no gubernamentales y de arreglos bilaterales entre gobiernos- a prestar apoyo a los paises en desarrollo en el mejoramiento de la producción de materiales y elementos de construcción por el sector no estructurado. En particular, se recomendó que la comunidad internacional:

a) Ayudara a difundir información sobre las normas de materiales de construcción aplicadas en diversos países, así como a formular normas y especificaciones para diversos materiales locales;

b) Prestara asistencia a los gobiernos en el fortalecimiento de las actividades de centros locales de adaptación de tecnología o - si fuera necesario- para el establecimiento de tales centros como puntos focales de redes de intercambio de tecnología entre paises en desarrollo y para la promoción de proyectos de demostración que incorporasen las tecnologías adaptadas;

c) Facilitara y estimulara la aplicación de "know-how" y la difusión de información sobre los métodos para la gestión de la producción y el empleo de materiales de construcción por el sector no estructurado, bien entendido que, entre los muchos medios de difusión, revestian especial imortancia los manuales sencillos ilustrados y los folletos fácilmente comprensibles, así como los proyectos de demostración;

d) Prestara asistencia a los gobiernos en la celebración de seminarios y jornadas de estudio sobre diversos temas de interés para los diversos grupos del sector no estructurado considerados como objetivo, y referentes a aspectos tales como la formulación de políticas, el desarrollo de programas, las actividades de capacitación y el mejoramiento de la tecnologia, concediendo prioridad a los materiales de construcción que interesen al sector no estructurado de los paises en desarrollo;

e) Tratara de realizar, mediante la colaboración entre organismos internacionales, un estudio sistemático de los datos existentes sobre la producción y el empleo de materiales y elementos de construcción en el sector no estructurado, estableciendo al mismo tiempo directrices para la reunión y el procesamiento de los datos, identificando las lagunas en materia de información y desarrollando instrumentos analíticos;

La Primera Consulta recomendó que la ONUDI y el Centro de las Naciones Unidas para los Asentamientos Humanos (Hábitat), en colaboración con otros organismos internacionales y regionales interesados, estudiaran las posibilidades de mejorar la cooperación regional en Africa, América Latina y Oriente Medio, con objeto de promover la cooperación entre países en desarrollo en la recopilación y el intercambio de información, la investigación y el desarrollo a nivel plurinacional, los servicios de consultoría y la capacitación en materia de fabricación y empleo de materiales y elementos de construcción baratos, con especial hincapié en el sector no estructurado.
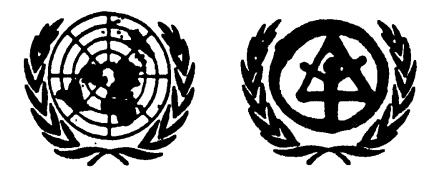